\title{
Inflammatory myofibroblastic tumor in the head of the pancreas with anorexia and vomiting in a 69-year-old man: A case report
}

\author{
DING DING ${ }^{1}$, XIANMIN BU $^{2}$ and FENG TIAN ${ }^{1}$ \\ Departments of ${ }^{1}$ Gastroenterology and ${ }^{2}$ Surgery, \\ Shengjing Hospital of China Medical University, Shenyang, Liaoning 110004, P.R. China
}

Received December 18, 2014; Accepted January 21, 2016

DOI: $10.3892 / \mathrm{ol} .2016 .4787$

\begin{abstract}
Inflammatory myofibroblastic tumor (IMT) is a rare condition of unclear etiology that is commonly observed in the lung but rarely in the pancreas. WHO classified IMT as a potentially malignant or aggressive tumor. In the present report, the case of a 69-year-old male patient with an IMT in the head of the pancreas, who experienced anorexia, nausea and vomiting, is presented. The patient's clinical symptoms were nonspecific, and the imaging findings revealed a hypovascularized pancreatic mass with stenosis of the descending duodenum. The electronic endoscopy findings revealed protruding lesions in the duodenal bulb and the descending duodenum. Biopsies of the mass were conducted with an electronic endoscope, but were not diagnostic. Subsequent duodenopancreatectomy aided in determining a pathological diagnosis of IMT, based on the histology and immunohistochemistry results. The patient experienced a recovery without further incident, as observed during a regular follow-up 3 years later. IMT in the head of the pancreas is rare, particularly in adults. In the present study, an extremely rare case of IMT involving the head of the pancreas in an adult patient is presented, and the therapeutic options for this condition are discussed.
\end{abstract}

\section{Introduction}

Inflammatory myofibroblastic tumor (IMT) is an uncommon type of mesenchymal tumor (1). The current World Health Organization (WHO) classification for this rare tumor entity

Correspondence to: Dr Feng Tian, Department of Gastroenterology, Shengjing Hospital of China Medical University, 36 Sanhao Street, Shenyang, Liaoning 110004, P.R. China E-mail: tianfeng@sj-hospital.org

Abbreviations: ALK, anaplastic lymphoma kinase; CEA, carcinoembryonic antigen; CT, computed tomography; H\&E, hematoxylin and eosin; IMT, inflammatory myofibroblastic tumor; SMA, smooth muscle actin; WHO, World Health Organization

Key words: head of pancreas, inflammatory myofibroblastic tumor, diagnosis, treatment is a fibroblastic sarcoma or myofibroblastoma, which is a distinctive neoplasm of intermediate biological potential that may be malignant or aggressive (2). The worldwide incidence of IMT is $0.04-0.7 \%(3,4)$ and clinical data has shown that IMTs have a $25 \%$ rate of local recurrence, and up to a $5 \%$ rate of distant metastasis (2).

IMT mostly occurs in visceral soft tissues, including the lungs, mesentery, omentum, retroperitoneum, pelvis and abdominal soft tissue (5). IMT mostly affects children and adolescents, while being scarcely observed in old people, and generally does not exhibit any gender preference (5). Pancreatic IMT is rare, however previous studies have shown that this type of occurrence may be more common in women $(6,7)$. A total of $60 \%$ of pancreatic IMTs are located in the head of the pancreas, while $40 \%$ of cases are located in the body and tail $(6,8)$. Surgery is the primary treatment for pancreatic IMT, and in rare cases this may be complemented by treatment with steroids and/or radiation $(7,9)$. The prognosis of the disease is generally favorable, and regular follow-up is necessary.

In the current study, the case of a 69-year-old man who presented to the Shengjing Hospital of China Medical University (Shenyang, China) with symptoms of anorexia, nausea and vomiting caused by an IMT in the head of the pancreas, is reported. Surgical resection was conducted, and the patient had a regular follow-up 3 years later. Written informed consent was obtained from the patient.

\section{Case report}

A 69-year-old man was admitted to the Shengjing Hospital of China Medical University on January 9, 2013 with a 3-month history of anorexia, upper abdominal distension and vomiting. Nausea and vomiting frequently occurred following a meal. Undigested food and bile were occasionally present in the vomitus. Pain, fever, jaundice and melena were not reported, although the patient had experienced a weight loss of $10 \mathrm{~kg}$. His medical history was unremarkable. Physical examination revealed absence of tenderness in the epigastric area. Succussion splash and Murphy's sign were negative, and the patient's bowel sounds were normal. Laboratory tests, including complete blood count, urinalysis, amylase test and lipase test, were normal. The levels of tumor markers, including $\alpha$-fetoprotein, $2.20 \mathrm{ng} / \mathrm{ml}$ (normal range, 0.00-9.00 ng/ml), carcinoembryonic antigen (CEA), $2.87 \mathrm{ng} / \mathrm{ml}$ (normal range, 0.00-5.00 ng/ml), 

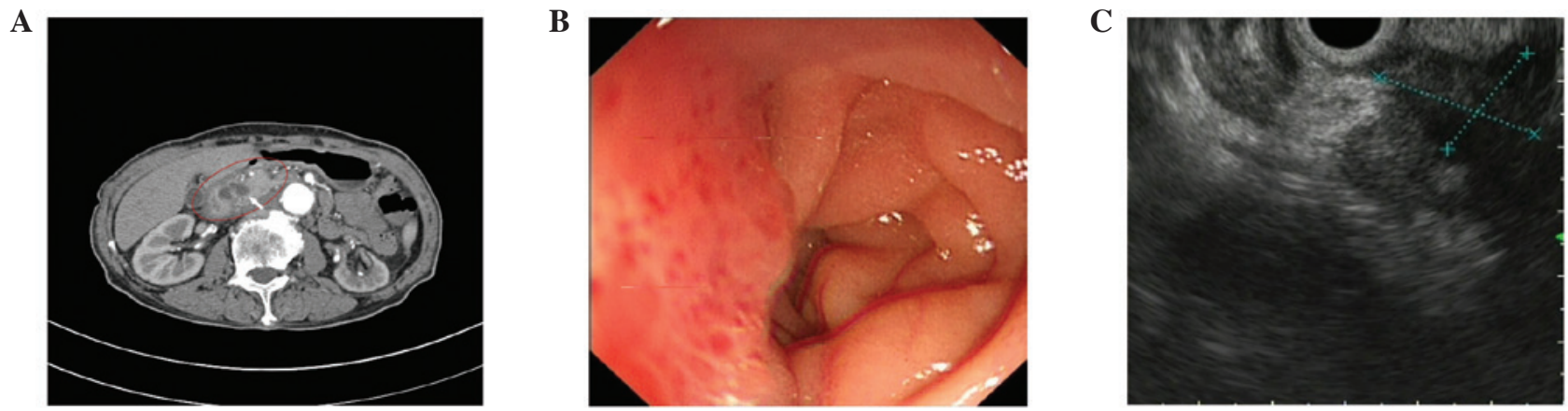

Figure 1. Enhanced abdominal CT, endoscopy and endoscopic ultrasound examination of the lesion. (A) Enhanced abdominal CT revealed an irregular low-density mass that extended into the head of the pancreas and duodenum (red circle). Tortuous and dilated lumens were observed in the mass (white arrow). (B) Endoscopy revealed an ulcerated, protruding lesion in the duodenal bulb and the descending duodenum. (C) Endoscopic ultrasound revealed a 23.0x19.0-mm unorganized mass with an ill-defined border that extended into the duodenal bulb and the descending duodenum. CT, computed tomography.

carbohydrate antigen (CA)19-9, $15.26 \mathrm{U} / \mathrm{ml}$ (normal range, $0.00-37.00 \mathrm{U} / \mathrm{ml}$ ) and $\mathrm{CA} 72-4,1.30 \mathrm{U} / \mathrm{ml}$ (normal range, 0.00-6.90 U/ml), were negative. Enhanced abdominal computed tomography (CT; SOMATOM Definition AS; Siemens Healthcare, Erlangen, Germany) examination revealed a cystic-solid tumor located between the pancreas and the duodenum (Fig. 1A). No evidence of distant organ or lymph node metastasis was observed. Endoscopy revealed a anabrotic protrusion lesion located between the duodenal bulb and the descendant duodenum (Fig. 1B). Endoscopic ultrasound (PENTAX EG-2970K and PENTAX EG-3870UTK; Pentax, Tokyo, Japan) examination revealed a 23.0x19.0-mm protrusive low-echo mass, which had unclear boundaries with the adjacent pancreas, located between the duodenal bulb and the descendant duodenum (Fig. 1C). The endoscopic biopsy of the mass was inconclusive, since it demonstrated a fibrous lesion containing inflammatory cells without any evidence of malignancy. Since malignant pancreatic head enlargement with duodenum metastasis was suspected, the patient underwent an operation in order to establish a diagnosis by operative pathological biopsy. During the operation, a large, hard mass with a diameter of $\sim 4.0 \times 3.0 \mathrm{~cm}$, which was located in the head of the pancreas and metastasized into the duodenum, was identified. The gross specimen was fleshy, with a white cut surface. Necrosis and hemorrhage were observed (Fig. 2). Histopathologically, the tumor cells were arranged mainly in a fusiform pattern with an inflammatory infiltrate of lymphocytes and eosinophils (Fig. 3). Samples were stained with hematoxylin and eosin (Sigma-Aldrich, St. Louis, MO, USA) and visualized using a microscope (Eclipse E800; Olympus Corporation, Tokyo, Japan). Immunohistochemical staining was performed with the following antibodies: mouse monoclonal anti-human smooth muscle actin (SMA; cat. no. ZM-0003; dilution, 1:50; Beijing Zhongshan Jinqiao Biological Technology Co., Ltd., Beijing, China), mouse monoclonal anti-human desmin (cat. no. ZM-0091; dilution, 1:50; Beijing Zhongshan Jinqiao Biological Technology Co., Ltd.), mouse monoclonal anti-human $\beta$-catenin (cat. no. ZM-0442; dilution, 1:50; Beijing Zhongshan Jinqiao Biological Technology Co., Ltd.), mouse monoclonal anti-human cluster of differentiation (CD)34 (cat. no. ZM-0046; dilution, 1:50; Beijing Zhongshan Jinqiao Biological Technology Co., Ltd.), mouse monoclonal anti-human CD117 (cat. no. ZM-0437;

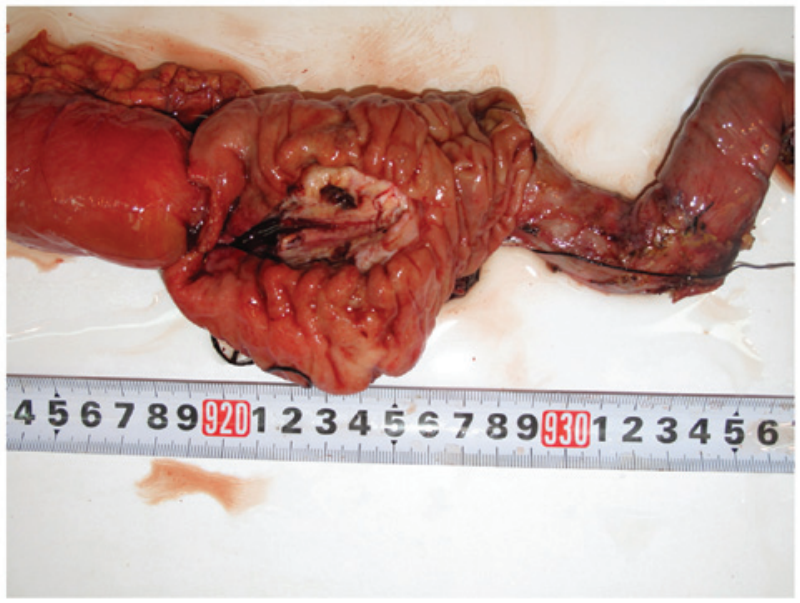

Figure 2. Photograph of the resected specimen revealing the macroscopic appearance of the fleshy, lobulated and circumscribed mass $(4.0 \times 3.0 \mathrm{~cm})$ with mixed solid and cystic components, which was located in the head of the pancreas. Necrosis and hemorrhage were observed.

dilution, 1:50; Beijing Zhongshan Jinqiao Biological Technology Co., Ltd.) and mouse monoclonal anti-human DOG-1 (cat. no. ZM-0371; dilution, 1:50; Beijing Zhongshan Jinqiao Biological Technology Co., Ltd.). Phosphate-buffered saline (Beijing Zhongshan Jinqiao Biological Technology Co., Ltd.) was used as the dilution buffer and tissue sections were incubated with the primary antibodies overnight at $4^{\circ} \mathrm{C}$. The sections were then washed twice and incubated with horseradish peroxidase-conjugated goat anti-mouse IgG secondary antibodies (cat. no. SP-9002; ready to use; Beijing Zhongshan Jinqiao Biological Technology Co., Ltd.) for $60 \mathrm{~min}$ at room temperature. Staining was visualized using a microscope (Eclipse E800; Olympus Corporation). Immunohistochemically, the tumor was positive for SMA (Fig. 4A), desmin (Fig. 4B) and $\beta$-catenin (Fig. 4C), but negative for CD34 (Fig. 4D), CD117 (Fig. 4E) and DOG-1 antibody (Fig. 4F). A pathological diagnosis of IMT was established based on the histology and immunohistochemistry results. The postoperative course was uneventful, and the patient was discharged from hospital on January 30, 2013. No adjuvant treatment was administered following surgical excision of the tumor, and no evidence of a recurrent tumor was observed during the 3 year follow-up. 
A

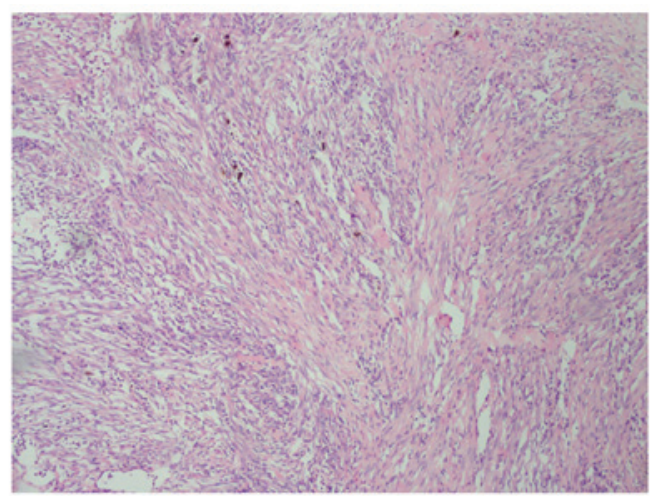

B

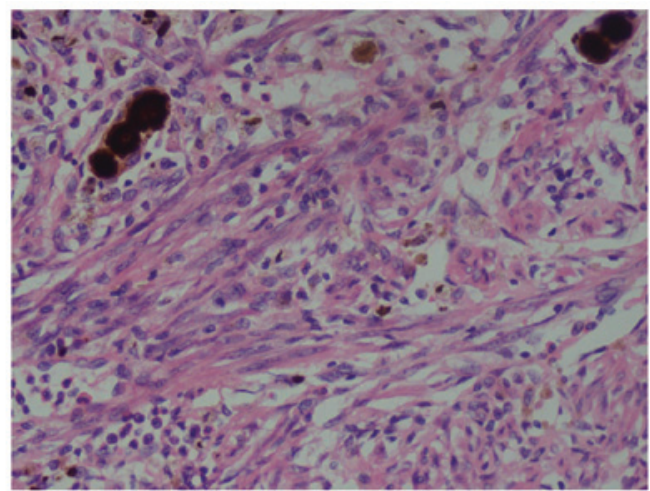

Figure 3. Photomicrograph of hematoxylin and eosin staining of the lesion. (A) A fusiform pattern with a variable amount of inflammatory infiltrate composed of lymphocytes and eosinophils in a myxoid matrix was observed. Magnification, x100. (B) Nuclear pleomorphism and mitotic figures were not prominent, but occasional atypical cells with large nuclei and necrotic cystic components were observed. Magnification, x200.

A

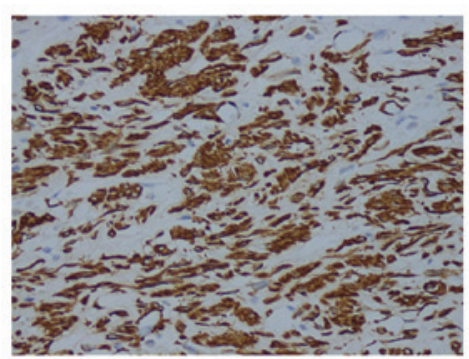

D

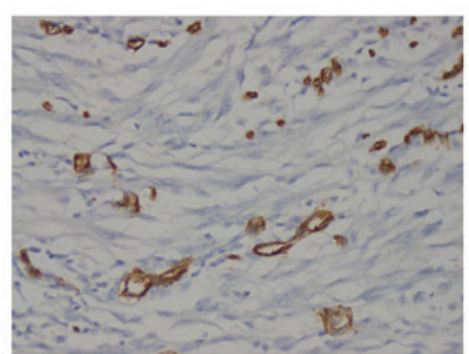

B

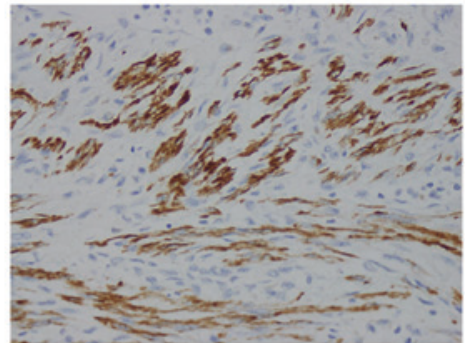

$\mathbf{E}$

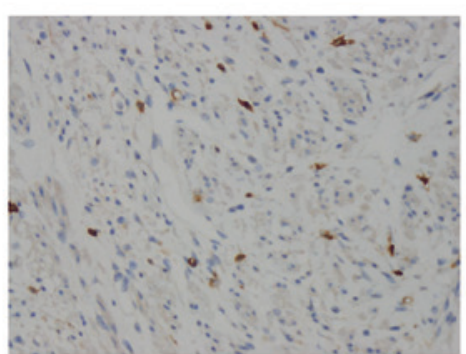

C

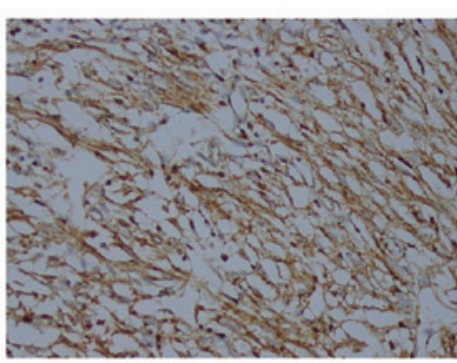

$\mathbf{F}$

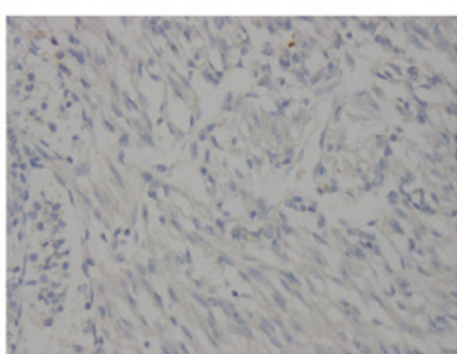

Figure 4. Photomicrograph of immunohistochemistry staining of the lesion. (A) Tumor cells were positive for smooth muscle actin. Smooth muscle of blood vessels acted as an internal positive control. Tumor cells were immunopositive for (B) desmin and (C) $\beta$-catenin, but immunonegative for (D) CD34. Endothelial cells of blood vessels were used as an internal positive control. Tumor cells were immunonegative for (E) CD117 and (F) DOG-1 antibody. Magnification, x200. $\mathrm{CD}$, cluster of differentiation.

\section{Discussion}

IMT, which is clinically rare, was considered to be a reactionary inflammatory lesion following trauma, surgery or infection, and was previously referred to as an inflammatory pseudotumor, plasma cell granuloma, xanthomatous pseudotumor, xanthoma or histiocytoma $(10,11)$. Subsequent studies have identified that IMT is a neoplasm with frequent clonal alterations in the chromosome $2 \mathrm{p} 23$ and with the potential for local aggressiveness, recurrence, metastasis, malignant transformation and myofibroblastic differentiation (11-13). The current WHO classification for this rare tumor entity is a fibroblastic sarcoma or myofibroblastoma, which is a distinctive neoplasm of intermediate biological potential that may be malignant or aggressive $(2,10,14)$. IMT principally affects young patients, and does not exhibit any gender preference. IMT may occur in the whole body, although the most common sites are the lungs, head, neck, extremities, intra-abdominal and retroperitoneal soft tissues $(10,11,15)$. To the best of our knowledge, a rare case of a pancreatic IMT that caused intestinal obstruction in an old man has been previously published (8). Despite being previously regarded as a reactive lesion, IMT is currently considered to be a neoplastic process $(10,11)$. Therefore, its correct diagnosis is critical for offering the patients the appropriate disease management and subsequent treatment.

The clinical presentation of IMT varies depending on its anatomic location and whether it involves vital organs (16). For patients with IMT, pancreatic involvement is often inconclusive, since patients may generally present with nonspecific symptoms such as abdominal pain, weight loss and jaundice (11). In rare cases, IMT may present with anemia, fatigue, sudden diabetes or a palpable mass that resembles pancreatic carcinoma, periampullary carcinoma, lymphoma and endocrine tumors (11). The radiological features of IMT are nonspecific, and exhibit 
variable characteristics (6). Ultrasound and CT examinations reveal a solid, or occasionally cystic-solid, mass in the pancreas, which is usually sized between 5.0 and $10.0 \mathrm{~cm}$ and may be well-demarcated or metastatic (7).

Due to its nonspecific symptoms and imaging findings, definitive diagnosis of IMT relies on histological evaluations (6). However, it is often difficult to obtain enough tissue using a transendoscopic biopsy to establish a definitive histological diagnosis (17). In the present patient, a gastroscopic biopsy of the lesion was inconclusive, and surgical resection of the tissues aided to obtain the final diagnosis of IMT.

The histology results of the present case revealed the presence of an independent cell type between smooth muscle cells and fibroblasts, in addition to mixed spindle cells scattered among a large number of inflammatory cells, mostly lymphocytes, plasma cells, organized cells, eosinophils and neutrophils (14). It has been previously reported that three histological patterns may coexist variably within the same tumor, namely myxoid/vascular, compact spindle cell and fibrous hypocellular (15,18-20). Immunohistochemically, the majority of cases of IMT express SMA, desmin and cytokeratin $(15,17,21)$. Anaplastic lymphoma kinase (ALK) immunostaining is detected in $40-60 \%$ of cases, and involves rearrangements or translocations of the ALK receptor tyrosine kinase on the chromosome 2 p23 $(16,21)$. In previous studies, the histological features of IMT did not correlate with its clinical behavior (2). In addition, the risk of malignant transformation or metastasis has been described in certain cases, and remains low $(<5 \%)(2)$. In the present case, the tumor exhibited a compact spindle cell histological pattern, and there were no obvious signs of malignancy in the histology. Immunohistochemical staining demonstrated the tumor to be $\mathrm{SMA}^{+}$, desmin ${ }^{+}$, $\beta$-catenin ${ }^{+}, \mathrm{CD}^{-} 4^{-}, \mathrm{CD}_{117}{ }^{-}$and DOG-1. During a regular 3 year follow-up, the patient was observed to be healthy.

Currently, despite the difficulty of obtaining a definitive diagnosis based on biopsies and radiological examinations, surgery with complete excision remains the primary therapeutic option for IMT, although no real consensus regarding the treatment of IMT exists $(7,9)$. The low risk of malignant transformation or metastasis has been described in previous cases of IMT, but if the excision is incomplete, the risk increases from $<5$ to $25 \%$ (2). Due to the possibility of malignancy and relapse, a number of authors have reported that a simple tumor excision is uncertain (8). Wide excision may be appropriate if the patient's physical condition is adequate $(7,9)$. However, whether radical resection for such intermediate, low-grade lesions is appropriate requires further clinical study. In the present report, the case of an elderly, infirm man who had IMT of the head of the pancreas with unclear boundaries with the adjacent duodenum, was presented. During surgery, no evidence of blood vessels, lymph node or other abdominal organs metastasis (except adjacent duodenum) was observed. In consequence, a duodenopancreatectomy was performed. For similar patients, a surgical approach on a case-by-case basis may be considered in the future. For non-excisable lesions, several medical treatments, including chemotherapy, radiation therapy, nonsteroidal anti-inflammatory drugs, corticosteroids and antitumor necrosis factor-binding antibodies, have been previously administered to palliate or shrink these IMTs to a resectable size and configuration $(6,7,9)$. However, the medical treatment is not clearly defined due to a lack of strong statistical evidence, and palliative treatment programs cannot be recommended as a standard regimen $(22,23)$. Based on the recent advances in ALK inhibitors and other ALK target genes, these therapies may be more effective, and thus may be offered to patients with IMT instead (16). Whether surgical resection or drug therapy is the selected treatment, the prognosis of the disease is favorable, with distant metastases occurring rarely, and a 5-year survival rate of $91 \%(10,11,15)$. However, close, long-term follow-up is necessary due to the probability of malignancy, distant metastasis and recrudescence exhibited by IMT (15).

In conclusion, in this study the rare case of pancreatic head IMT in a 69-year-old man, with aggressive behavior and metastasis to the duodenum was presented. However, the patient's clinical symptoms and imaging findings were nonspecific and diagnosis relied on histopathological examination and immunohistochemistry. Complete surgical resection is the preferred treatment for IMT. The prognosis of the disease is generally favorable, and regular follow-up is necessary.

\section{References}

1. Patnana M, Sevrukov AB, Elsayes KM, Viswanathan C, Lubner $\mathrm{M}$ and Menias CO: Inflammatory pseudotumor: The great mimicker. AJR Am J Roentgenol 198: W217-W227, 2012.

2. Fletcher CDM, Bridge JA, Hogendoorn P and Mertens F (eds): World Health Organization Classification of Tumours of Soft Tissue and Bone. In: World Health Organization Classification of Tumors. Vol 5. 4th edition. IARC Press, Lyon, 2013.

3. Pinilla I, Herrero Y, Torres MI, Nistal M and Pardo M: Myofibroblastic inflammatory tumor of the lung. Radiologia 49: 53-55, 2007 (In Spanish).

4. Panagiotopoulos N, Patrini D, Gvinianidze L, Woo WL, Borg E and Lawrence D: Inflammatory myofibroblastic tumour of the lung: A reactive lesion or a true neoplasm? J Thoracic Dis 7: 908-911, 2015.

5. Zhang T, Yuan Y, Ren C, DU S, Chen J, Sun Q and Liu Z: Recurrent inflammatory myofibroblastic tumor of the inguinal region: A case report and review of the literature. Oncol Lett 10: 675-680, 2015.

6. Sim A, Lee MW and Nguyen GK: Inflammatory myofibroblastic tumour of the pancreas. Can J Surg 51, E23-E24, 2008.

7. Dagash H,Koh C, Cohen M, Sprigg A and Walker J: Inflammatory myofibroblastic tumor of the pancreas: A case report of 2 pediatric cases - steroids or surgery? J Pediatr Surg 44: 1839-1841, 2009.

8. Pungpapong S, Geiger XJ and Raimondo M: Inflammatory myofibroblastic tumor presenting as a pancreatic mass: A case report and review of the literature. JOP 5: 360-367, 2004.

9. Tomazic A, Gvardijancic D, Maucec J and Homan M: Inflammatory myofibroblastic tumor of the pancreatic head - a case report of a 6 months old child and review of the literature. Radiol Oncol 49: 265-270, 2015.

10. Fragoso AC, Eloy C, Estevão-Costa J, Campos M, Farinha N and Lopes JM: Abdominal inflammatory myofibroblastic tumor a clinicopathologic study with reappraisal of biologic behavior. J Pediatr Surg 46: 2076-2082, 2011.

11. Gleason BC and Hornick JL: Inflammatory myofibroblastic tumours: Where are we now? J Clin Pathol 61: 428-437, 2008.

12. Ernst CW, Van Der Werff Ten Bosch J, Desprechins B, de Mey J and De Maeseneer M: Malignant transformation of an abdominal inflammatory myofibroblastic tumor with distant metastases in a child. JBR-BTR 94: 78-80, 2011.

13. Fisher C: Myofibroblastic malignancies. Adv Anat Pathol 11: 190-201, 2004.

14. Coindre JM: New WHO classification of tumours of soft tissue and bone. Ann Pathol 32 (Suppl 5): S115-S116, 2012 (In French).

15. Coffin CM, Watterson J, Priest JR and Dehner LP: Extrapulmonary inflammatory myofibroblastic tumor (inflammatory pseudotumor). A clinicopathologic and immunohistochemical study of 84 cases. Am J Surg Pathol 19: 859-872, 1995.

16. Butrynski JE, D'Adamo DR, Hornick JL, Dal Cin P, Antonescu CR, Jhanwar SC, Ladanyi M, Capelletti M, Rodig SJ, Ramaiya N, et al: Crizotinib in ALK-rearranged inflammatory myofibroblastic tumor. N Engl J Med 363: 1727-1733, 2010. 
17. Khakural P, Sapkota R, Shrestha UK and Sayami P: Successful surgical management of a rare esophageal inflammatory myofibroblastic tumour: A case report. J Cardiothorac Surg 10: $112,2015$.

18. Chan JK, Cheuk W and Shimizu M: Anaplastic lymphoma kinase expression in inflammatory pseudotumors. Am J Surg Pathol 25: 761-768, 2001.

19. Hussong JW, Brown M, Perkins SL, Dehner LP and Coffin CM: Comparison of DNA ploidy, histologic and immunohistochemical findings with clinical outcome in inflammatory myofibroblastic tumors. Mod Pathol 12: 279-286, 1999.

20. Makhlouf HR and Sobin LH: Inflammatory myofibroblastic tumors (inflammatory pseudotumors) of the gastrointestinal tract: How closely are they related to inflammatory fibroid polyps? Hum Pathol 33: 307-315, 2002.
21. Cook JR, Dehner LP, Collins MH, Ma Z, Morris SW, Coffin CM and Hill DA: Anaplastic lymphoma kinase (ALK) expression in the inflammatory myofibroblastic tumor: A comparative immunohistochemical study. Am J Surg Pathol 25: 1364-1371, 2001.

22. Germanidis G, Xanthakis I, Tsitouridis I, Zaramboukas T, Kiskinis D, Konstantaras C, Miliaras S, Sirakos T and Pagkalos E: Regression of inflammatory myofibroblastic tumor of the gastrointestinal tract under infliximab treatment. Dig Dis Sci 50: 262-265, 2005.

23. Applebaum H, Kieran MW, Cripe TP, Coffin CM, Collins MH, Kaipainen A, Laforme A and Shamberger RC: The rationale for nonsteroidal anti-inflammatory drug therapy for inflammatory myofibroblastic tumors: A Children's Oncology Group study. J Pediatr Surg 40: 999-1003, 2005. 